\title{
Frequency Oscillations in the Delta Scuti Star V534 Tauri: Preliminary Results of the STEPHI IX Campaign
}

\author{
L. Fox Machado ${ }^{1}$, Z. P. Li ${ }^{2}$, E. Michel ${ }^{3}$, M. Alvarez ${ }^{5}$, \\ M. M. Hernández ${ }^{1}$, M. Chevreton ${ }^{4}$, A. Zhou ${ }^{2}$, C. Barban ${ }^{3}$, \\ N. Dolez ${ }^{6}$, J. A. Belmonte ${ }^{1}$, A. Fernández ${ }^{4}$, J. Fremy ${ }^{4}$, S. Pau ${ }^{4}$,

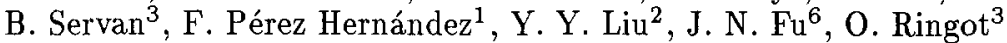

\section{Introduction}

The $\delta$ Scuti stars are pulsating variables located in the lower part of the Cepheid instability strip with spectral types from A2 to $\mathrm{F} 0$ on the main sequence, and from A3 to F5 at luminosity class III. These variables show short periods ( $<0.3 \mathrm{day})$ and luminosity amplitudes ranging from a few thousandths of a magnitude to several tenths. Over the last few years, significant progress has been made in the detection of pulsating modes in the framework of the multisite campaigns, e.g. STACC (Frandsen et al. 1996), DSN (Breger et al. 1998), STEPHI (Michel et al. 2000). For the 1998 STEPHI IX photometry campaign, the $\delta$ Scuti star V534 Tau of the Pleiades cluster (see Table 1) was monitored during a three week, three continent run. Preliminary results are reported here.

Table 1. Observational properties of the target stars in STEPHI IX

\begin{tabular}{lccccccc}
\hline Star & HD & Sp. & $m_{\mathrm{v}}$ & $B-V$ & $U-B$ & $\begin{array}{c}V \sin i \\
\left(\mathrm{~km} \mathrm{~s}^{-1}\right)\end{array}$ & $\beta$ \\
\hline V534 Tau & 23567 & A9V & 8.30 & +.36 & +.12 & 90 & 2.788 \\
Comparison 1 & 23628 & A4V & 7.68 & +.22 & +.10 & 187 & 2.884 \\
Comparison 2 & 23627 & B8 & 8.73 & +.18 & -.05 & & 2.804 \\
\hline
\end{tabular}

\section{Observations, Data Reduction and Modes Detected}

As was done in the other STEPHI campaigns, we observed from three observatories approximately equally-spaced in longitude: San Pedro Mártir Observatory in Baja California, Mexico; Xing Long Station in Beijing, China; and Teide Observatory in Tenerife, Spain. The observational procedure and the data reduction were the same as in the previous campaigns (see Alvarez et al. 1998).

\footnotetext{
${ }^{1}$ Instituto de Astrofísica de Canarias, E-38200 La Laguna, Tenerife, Spain

${ }^{2}$ Beijing Observatory, Chinese Academy of Sciences, Beijing, P.R. China

${ }^{3}$ Observatoire de Paris, DASGAL, URA CNRS 335, F-92195 Meudon, France

${ }^{4}$ Observatoire de Paris, DAEC, F-92195 Meudon, France

${ }^{5}$ Instituto de Astronomía-UNAM, Ap.P. 877, Ensenada, BC, México

${ }^{6}$ Observatoire Midi-Pyrénées, F-31400 Toulouse, France
} 
The amplitude spectrum of the time series of the target star was obtained by using the ISWF technique following the same procedure as that used in the STEPHI VII campaign (Hernández et al. 1998). The frequencies have been detected by using the prewhitening process. As is explained in Alvarez et al. (1998), the frequency peaks above a $99 \%$ confidence level are selected and subtracted iteratively from the original time series until the whole spectrum is below this level. Results are shown in Table 2.

\section{Conclusions}

Preliminary results of the STEPHI 98 campaign are reported. Eleven frequencies for the Delta Scuti star V534 Tau have been detected. A more complete study is in preparation ( $\mathrm{Li}$ et al., to be submitted to $\mathrm{A} \& \mathrm{~A}$ ). Information about the STEPHI network can be found at http://dasgal.obspm.fr/“stephi.

Table 2. Modes detected in our target star above a $99 \%$ confidence level. The resolution is $\Delta \nu \simeq 0.60 \mu \mathrm{Hz}$.

\begin{tabular}{lllccrc}
\hline Star & & $\begin{array}{c}\nu \\
(\mu \mathrm{Hz})\end{array}$ & $\begin{array}{c}\text { period } \\
(\text { hours })\end{array}$ & $\begin{array}{c}A \\
(\mathrm{mmag})\end{array}$ & $\begin{array}{c}\varphi \\
(\mathrm{rad})\end{array}$ & $\mathrm{S} / \mathrm{N}^{c}$ \\
\hline V534 Tau & $\nu_{1}$ & 448.060 & 0.619 & 2.130 & 0.846 & 15.8 \\
& $\nu_{2}$ & $377.857^{a, b}$ & 0.735 & 1.990 & -3.074 & 10.2 \\
& $\nu_{3}$ & 524.963 & 0.529 & 1.893 & 1.560 & 16.5 \\
& $\nu_{4}$ & 252.894 & 1.098 & 1.606 & 2.522 & 7.4 \\
& $\nu_{5}$ & 126.063 & 2.203 & 1.250 & 1.008 & 4.9 \\
& $\nu_{6}$ & $376.606^{a}$ & 0.737 & 1.250 & 2.070 & 6.4 \\
& $\nu_{7}$ & 234.217 & 1.185 & 1.168 & 0.128 & 5.1 \\
& $\nu_{8}$ & 191.836 & 1.447 & 1.124 & 2.189 & 4.8 \\
& $\nu_{9}$ & $379.026^{b}$ & 0.732 & 1.105 & -0.044 & 5.6 \\
& $\nu_{10}$ & 307.590 & 0.903 & 1.033 & -2.884 & 4.6 \\
& $\nu_{11}$ & 488.765 & 0.568 & 0.511 & -0.185 & 4.2 \\
\hline
\end{tabular}

${ }^{a}$ Pair of close frequencies (separation of $1.25 \mu \mathrm{Hz}$ ). ${ }^{b}$ Pair of close frequencies (separation of $1.16 \mu \mathrm{Hz}$ ). ${ }^{c}$ Signal-to-noise ratio.

\section{References}

Alvarez, M., Hernández, M. M., Michel, E., et al. 1998, A\&A, 340, 149

Breger, M., Zima, W., Handler, G., et al. 1998, A\&A, 331, 271

Frandsen, S, Balona, L. A., Viskum, M., et al. 1996, A\&A, 308, 132

Hernández, M. M., Michel, E., Belmonte, J. A., et al. 1998, A\&A, 337, 198

Michel, E., Chevreton, M., Belmonte, J. A., et al. 2000, in these proceedings, p. 483 